Ann. Biol. anim. Bioch. Biophys., I972, 12 (3), 505-508.

NOTE

\title{
INFLUENCE DE L'ACIDE ÉRUGIQUE ALIMENTAIRE CHEZ LA POULE PONDEUSE. DIMINUTION DU POIDS DE L'EUF
}

\author{
B. LECLERCQ \\ avec la collaboration technique de Marie-Rose Salichon \\ Station de Recherches avicoles, \\ Centre de Recherches de Tours, I. N. R. A., \\ 37 - Nousilly \\ RÉSUMÉ
}

L'incorporation au régime de la Poule pondeuse de 2 p. Ioo de triérucine provoque une réduction du poids de l'œuf et du vitellus équivalente à celle constatée avec $5 \mathrm{p}$. too d'huile de colza. L'acide érucique semble donc bien responsable des effets néfastes de l'huile de colza sur la reproduction de la Poule pondeuse.

\section{INTRODUCTION}

Plusieurs auteurs ont décrit simultanément les effets de l'huile de colza sur la reproduction de la Poule pondeuse (Kondra et Sell, I968; Leclerce, I968). Aux taux d'incorporation inférieurs à Io $\mathrm{p}$. Ioo, on ne constate en général aucune réduction de l'intensité de ponte (LEcLERco, I968). L'effet le plus manifeste et le plus constant concerne la réduction du poids moyen de l'œuf qui peut être provoquée par des doses très faibles d'huile de colza (LECLERCQ, 1970). Nous avons montré que ce phénomène n'apparaît pas avec 1'huile de canbra, qui est une huile de colza très pauvre en acide érucique ( $\mathrm{I}$ p. IOO), (LECLERCQ, I970). Cette observation suggère que l'acide érucique de l'huile soit responsable de la réduction du poids de l'œuf. Toutefois, la démonstration n'est pas pleinement satisfaisante, car rien n'empêche que d'autres substances accompagnant l'acide érucique soient responsables du phénomène observé.

Dans la présente expérience, nous nous proposons donc de comparer les effets d'un apport alimentaire équivalent d’acide érucique sous forme de triérucine, ou sous forme d'huile de colza. 


\section{MATÉRIEL ETT MÉTHODES}

On constitue trois lots homogènes de 6 poules issues d'un croisement Rhode Island $\times$ Leghorn (SykEs) et âgées de 45 semaines. Les animaux sont placés en cages individuelles, nourris et abreuvés à volonté.

L'essai comporte trois périodes de 28 jours. Au cours de la période préexpérimentale, les poules reçoivent le régime témoin (dépourvu d'acide érucique) ; cette période sert de référence pour chaque animal et permet de s'assurer de l'homogénéité des lots du point de vue de la ponte et du poids de l'œuf. Vient ensuite la période expérimentale pendant laquelle l'un des lots continue d'ingérer le régime témoin tandis que deux autres reçoivent les régimes enrichis, soit en triérucine, soit en huile de colza. Enfin, l'essai se termine par la période post-expérimentale au cours de laquelle toutes les poules reçoivent de nouveau le régime témoin.

La composition des régimes fait l'objet du tableau $\mathrm{x}$. Elle est aussi semblable que possible, si ce n'est pour l'apport de matières grasses qui est réalisé avec un apport simultané de cellulose

TABLEAU I

\section{Composition des régimes}

\begin{tabular}{|c|c|c|c|}
\hline & $\begin{array}{l}\text { Aliment } \\
\text { témoin }\end{array}$ & $\begin{array}{c}\text { Aliment } \\
\text { huile de colza }\end{array}$ & $\begin{array}{l}\text { Aliment } \\
\text { triérucine }\end{array}$ \\
\hline 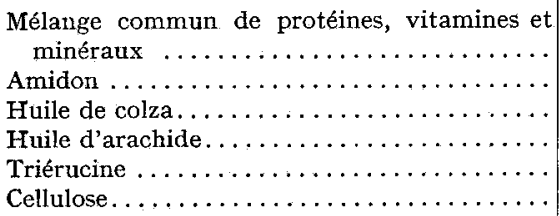 & $\begin{array}{l}73 \\
25 \\
- \\
- \\
-\end{array}$ & $\begin{array}{r}73 \\
15 \\
5 \\
-- \\
7\end{array}$ & $\begin{array}{r}73 \\
15 \\
- \\
3 \\
2 \\
7\end{array}$ \\
\hline
\end{tabular}

Composition du mélange commun

(pour roo $\mathrm{kg}$ )

\begin{tabular}{|c|c|c|c|c|}
\hline Farine de soja & $16,50 \mathrm{~kg}$ & Phosphate bicalcique ... & \multicolumn{2}{|c|}{$3,75 \mathrm{~kg}$} \\
\hline Farine de poisson...... & $2.75 \mathrm{~kg}$ & Chlorure de sodium ........ & \multicolumn{2}{|c|}{$0,25 \mathrm{~kg}$} \\
\hline Gluten de maïs ....... & $15,10 \mathrm{~kg}$ & Bicarbonate de sodium ...... & \multicolumn{2}{|c|}{$0,40 \mathrm{~kg}$} \\
\hline Huile de maïs . . . . . . . . & $1,35 \mathrm{~kg}$ & Carbonate de calcium & \multicolumn{2}{|c|}{$6,15 \mathrm{~kg}$} \\
\hline Farine de luzerne ...... & $10,90 \mathrm{~kg}$ & Amidon de mais ....... & \multicolumn{2}{|c|}{$42,50 \mathrm{~kg}$} \\
\hline Vitamine A & $1190000 \mathrm{UI}$ & Carbonate de magnésium .... & 16 & $\mathrm{~g}$ \\
\hline Vitamine $\mathrm{D}_{3} \ldots \ldots \ldots$ & $200000 \mathrm{UI}$ & Oxyde de magnésium ...... & 20 & $\mathrm{~g}$ \\
\hline Riboflavine $\ldots \ldots \ldots$ & $1 \mathrm{~g}$ & Sulfate de fer $\ldots \ldots \ldots \ldots \ldots$ & 15 & $g$ \\
\hline Vitamine $\mathrm{E}, \ldots \ldots \ldots$ & $4 \mathrm{~g}$ & Sulfate de zinc $\ldots \ldots \ldots \ldots$ & 30 & $\mathrm{~g}$ \\
\hline Chlorure de choline .... & $75 \mathrm{~g}$ & Sulfate de manganèse ...... & 40 & $\mathrm{~g}$ \\
\hline DL méthionine...$\cdots$ & $70 \mathrm{~g}$ & Sulfate de cuivre $\ldots \ldots \ldots \ldots$ & & \\
\hline
\end{tabular}

au détriment de l'amidon de façon à obtenir des teneurs en énergie métabolisable équivalentes. La composition de l'huile de colza a été rapportée dans une précédente publication (LECLERCQ, I970) ; la proportion d'acide érucique est de 49,6 p. Ioo. Les acides gras de la " triérucine "renferment 92 p. roo d'acide érucique et des traces d'acides palmitique, stéarique, oléique, linoléique, arachidique, gadoléique et béhénique. La " triérucine " est constituée de triglycérides purs obtenus par estérification du glycérol. 
Durant les I 4 derniers jours de chaque période, on effectue différentes mesures : nombre d'œufs, poids de l'œuf entier et poids du jaune, consommation d'aliment.

L'analyse statistique porte sur les différences entre les données de la période considérée et celles de la période de référence (période préexpérimentale).

\section{RÉSULTATS ET DISCUSSION}

Disposant d'une quantité très limitée de triérucine pure, nous avons dû limiter à six le nombre d'animaux par lot. De ce fait, il est impossible de tirer des conclusions statistiquement valables à propos du rythme de ponte et de la consommation. Néanmoins, ces deux critères varient peu. Il semble donc qu'aux faibles taux d'incorporation, l'acide érucique n'ait pas d'effet sur le rythme de ponte (LECLERCQ, I968).

Il est en tout autrement du poids de l'œuf entier et de celui du jaune; nous présentons ces résultats dans le tableau 2. La présence d'environ 2 p. roo d'acide érucique dans le régime de la

\section{TABIEAU 2}

Variations du poids de l'ceuf entier et du vitellus en fonction des régimes

Les variations accompagnées de la même lettre ne sont pas significativement différentes au seuil de signification de 1 p. Ioo

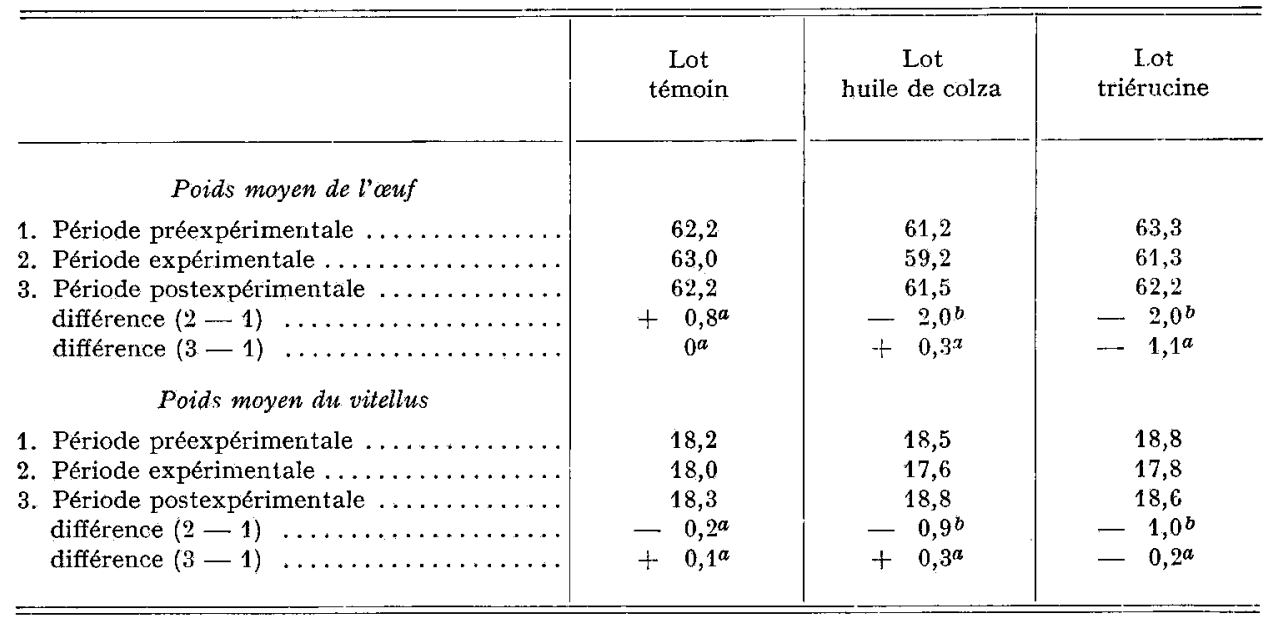

poule pondeuse conduit à une réduction du poids de l'œuf de 2 grammes, que l'acide érucique provienne de 1'huile de colza ou de la triérucine. Comme, parallèlement, dans le lot témoin, le poids moyen de l'œuf augmente de o,8 gramme, l'effet dépressif de l'acide érucique peut être chiffré à 2,8 grammes. De même, le poids du vitellus diminue d'environ $\mathbf{r}$ gramme.

Le retour à un régime dépourvu d'acide érucique (période post-expérimentale) permet d'effacer presque totalement les différences entre lots. Il subsiste, cependant, un léger retard dans le lot recevant de la triérucine, bien que cette différence ne soit pas significative.

On peut admettre que les acides gras présents à l'état de traces dans la " triérucine " n'ont pas d'effet sur la vitellogenèse. Il en est ainsi des acides palmitique, stéarique, oléique et linoléique qui ont fait déjà l'objet de nombreuses études. Par ailleurs, on peut penser que les acides gado- 
léique ( 2 p. 10o) et béhénique ( $\mathbf{~ p . ~ 1 0 o ) ~ a y a n t ~ u n e ~ s t r u c t u r e ~ v o i s i n e ~ d e ~ l ' a c i d e ~ e ́ r u c i q u e ~ n e ~ p e u v e n t ~}$ avoir qu'une activité analogue. La disproportion entre les apports de ce dernier et des deux autres permet d'envisager raisonnablement que l'acide érucique est bien le principal agent actif de la "triérucine".

Dans ces conditions, nos résultats ainsi que ceux acquis précédemment avec l'huile de colza très pauvre en acide érucique (LECLERCQ, 1970) ont tout lieu de faire croire que l'acide érucique est responsable des effets néfastes constatés chez la poule pondeuse. Il ne semble pas qu'il agisse au niveu digestif. La valeur énergétique exprineée en calories métabolisables est la même pour l'huile de colza que pour les autres huiles végétales (SELl et HodGson, 1962 ; Joshi et SELL, I964). Par ailleurs, la digestibilité apparente de l'acide érucique est élevée; de même, l'utilisation de l'acide érucique marqué avec le carbone $\mathrm{I}_{4}$ et ingéré par voie orale confirme que cet acide gras franchit aisément la barrière intestinale (LECLERCQ, non publié).

On doit donc penser que l'acide érucique intervient au niveau métabolique. Les mécanismes de cette intervention sont à démontrer. Il faut toutefois rapprocher les résultats concernant l'huile de colza de ceux obtenus avec les huiles de poisson (Roland et Edwards, 1967). Ces deux matières grasses ont comme caractéristique commune de contenir des acides gras à chaîne très longue (plus de 20 carbones). On est en droit de se demander si de tels acides gras ne possèdent pas des propriétés métaboliques particulières qui expliqueraient leur effet dépressif sur la reproduction de la poule.

Reçu pour publication en février 1972.

\section{REMERCIEMENTS}

Nous remercions la société Astra-Calvé qui nous a fourni la triérucine.

\section{SUMMARY}

\section{EFFECT OF ERUCIC ACID IN FEEDS ON EGG WEIGHT DECREASE IN LAYERS}

Identical weight decrease occurs in the egg and the yolk when either $2 \mathrm{p}$. roo trierucine or $5 \mathrm{p}$. Ioo rapeseed oil is added to the diet of layers. Erucic acid thus seems to be the factor responsible for the depressive effects of rapeseed oil.

Erucic acid is well digested. Its action probably occurs during metabolism. These results are similar to those on layers obtained with very long fatty acids in fish oil (RoLAND and EDwARDs, 1967).

\section{RÉFÉRENCES BIBLIOGRAPHIQUES}

Joshi S. K., SelL J. L., r964. Comparative dietary value of soybean oil, sunflower oil, rapeseed oil and animal tallow for turkey poults. Canad. J. anim. Sci., 44, 34-38.

Kondra P. A., Choo S. H., Sell J. L., Ig68. Influence of strain of chicken and dietary fat on egg production traits. Poult. Sci., 57, I290-I 296.

LECLERCQ B., I968. Utilisation de l'huile de colza par la poule pondeuse; son influence sur la genèse et la composition des lipides de l'œuf. C, R. Acad. Sci., 267, 223-2237.

LecLerco B., r97o. Facteurs nutritionnels modifiant le poids de l'œuf et de ses constituants. Ann. Biol. anim. Bioch. Biophys., 10, 239-252.

Rolands D. A., EDwards H. M., r967. Performance of laying hens fed carbohydrate free diets. Poult. Sci., 56, 1312.

:Sell J. L., Hodgson G. C., I962. Comparative value of dietary rapeseed oil, sunflower oil, soybean oil, and animal tallow for chickens. J. Nutr., Y6, I $13-118$. 